\title{
Building Evidence-Based Tobacco Treatment in the Eastern Mediterranean Region: Lessons Learned by the Syrian Center for Tobacco Studies
}

\author{
Taghrid Asfar, ${ }^{1,2}$ Kenneth D. Ward, ${ }^{2,3}$ Radwan Al-Ali, $^{2}$ and Wasim Maziak ${ }^{2,4}$ \\ ${ }^{1}$ Department of Public Health Sciences, University of Miami Miller School of Medicine, Miami, FL \\ 2 Syrian Center for Tobacco Studies, Aleppo, Syria \\ ${ }^{3}$ Division of Social and Behavioral Sciences, School of Public Health, University of Memphis, Memphis, TN \\ ${ }^{4}$ Department of Epidemiology, Robert Stempel College of Public Health and Social Work, Florida International University, \\ Miami, FL
}

\begin{abstract}
T he tobacco epidemic in Syria is characterised by high rates of cigarettes smoking in men and dramatic re-emergence of waterpipe smoking, especially among youths and women. The Syrian Center for Tobacco Studies (SCTS), an NIH-funded pioneer research and capacity building institution, has developed a research infrastructure and conducted three randomised clinical trials to develop and rigorously test culturally appropriate tobacco treatment programmes integrated into primary healthcare $(\mathrm{PHC})$ centres. This review aimed to discuss challenges and lessons learned from the Syrian experience. Addressing these challenges may inform future cessation research activities in Syria and other developing countries. To develop a research infrastructure, the SCTS has established Syria's first IRB and trained physicians/medical students in both tobacco treatment and research methods. Main challenges to conduct the cessation trials were difficulties of coordination between the local and international collaborators; high Smoking Rates among PHC providers; lack of pharmacological agents used in tobacco treatment; and difficulties of conducting research in a politically volatile region. Strategies to overcome these challenges were ensuring an active and regular involvement of all investigators; and advocating for a national smoking cessation plan that involves training healthcare providers in smoking cessation treatment and make pharmacological agents used in smoking cessation available.
\end{abstract}

\section{Introduction}

The tobacco epidemic is one of the major public health threats the world has ever faced, killing around 6 million people every year (WHO, 2008). Nearly $80 \%$ of smokers worldwide live in low- and middle-income countries, where the burden of tobacco-related illness and death is heaviest (WHO, 2008). Most smokers are interested in quitting and both behavioural counselling and pharmacotherapy can more than double the chances of quitting successfully (Fiore et al., 2008). However, in most lowincome countries, including Syria, smoking cessation services are still not available and studies looking at cessation and treatment options continue to lag behind (Maziak, Eissenberg, Klesges, Keil, \& Ward, 2004a; WHO, 2008).
Syria is a low-middle income country on the Eastern shore of the Mediterranean Sea and home to roughly 22 million inhabitants from diverse religious and ethnic backgrounds. Beginning in 2000, data on tobacco use in Syria began to emerge demonstrating high rates of tobacco use with about $51 \%$ of men, and $12 \%$ of women smoked cigarettes regularly. Beyond cigarettes, use of the waterpipe (also known as narghil, hookah, or sheesha) is growing alarmingly in Syria, particularly among women and youths (Asfar, Ward, Eissenberg, \& Maziak, 2005; Ward et al., 2006). Research indicates that more than $20 \%$ of men and $5 \%$ of women smoked waterpipe, and $14 \%$ of men and $3 \%$ of women smoked both waterpipe and cigarette (Maziak, 2002; Ward et al., 2006). Beside the high smoking

Address for correspondence: Taghrid Asfar, MD, MSPH, Department of Public Health Sciences, University of Miami Miller School of Medicine, Clinical Research Building, 1120 NW 14th Street, Miami,FL 33136.Email: tasfar@miami.edu 
rates in Syria, Syrian cigarettes smokers are less successful at quitting than smokers in developed countries (Control \& Prevention, 2011; Maziak et al., 2004a; Ward et al., 2005). This lack of success can be attributable mainly to the lack of support for cessation as pharmacological agents such as NRT, or bupropion (Zyban) are not sold in Syria, and behavioural counselling services do not exist (Maziak, 2002; Maziak et al., 2004a).

In Syria, as many other developing nations, clinical practice standards for smoking cessation are not in place, little work is being done to develop culturally appropriate interventions, and the transferability of effective interventions from more developed nations has not been tested. More importantly, most existing cessation programmes do not take into account regional tobacco use methods such as waterpipe smoking. These deficits have been exacerbated considerably by the Syrian civil war which has been on-going since 2011 (The war on Syrian civilians, 2014). In this review, we described challenges and lessons learned from the Syrian experience in developing and testing culturally appropriate and evidence-based tobacco treatment programmes integrated in PHC centres, we also provided future directions for smoking cessation efforts in Syria and other developing countries.

\section{Establishment of the Syrian Center for Tobacco Studies}

As we have mentioned above, given the high rates of tobacco use and lack of empirically based research, prevention, treatment, or training in Syria, there was a great need for infrastructure and training. In 2002, with the support of the National Institute of Health's Fogarty International Center and its partners (including NIDA, IC Health, IDRC, and EU), the SSCTS (www.scst-sy.org) was established to address the tobacco epidemic in Syria (Maziak, Ward, Eissenberg, Klesges, \& Keil, 2004c). The main objectives of the SCTS were to: (1) establish local scientific base in tobacco research by building a research infrastructure through partnerships among scientific institutions from developed and developing countries, and (2) develop and test culturally appropriate, efficacious, and cost-effective smoking cessation interventions that can be integrated in PHC centres in Syria (Maziak et al., 2004a; Richmond \& Heather, 1990). Our rationale was that developing capacity to deliver and evaluate smoking cessation interventions is a logical starting point to improve Syria's tobacco control infrastructure. Helping smokers quit is essential to reducing tobacco-related morbidity and mortality in the short-term (Jha \& Chaloupka, 2000). Realising this, at the outset of our work the Syrian government was highly supportive of our efforts and the national Ministry of Health served as a partner. Achieving these objectives, however, required confronting some initial serious challenges. Below we discuss these challenges and strategies used by the SCTS to overcome these challenges.

\section{Challenges for Building a Research Infrastructure in Tobacco} Control in Syria

Challenges encountered in building a research infrastructure were the absent of Institutional Review Board in the country, and lack of experience in research methods.

The absence of institutional review board. At the inception of the SCTS, no Institutional Review Board was located in Syria, nor did any Syrian institutions have human subjects protection agreements with U.S. funders, such as a federal wide assurance. Thus, first steps in forming the SCTS was to ensure that this institute acted in accordance with international standards for research involving human subjects, by obtaining a federalwide assurance and establishing Syria's first IRB. We trained IRB members in research ethics and their role/authorities and provided ongoing consultation (Maziak, Arora, Reddy, \& Mao, 2006). In addition, all researchers and staff were trained in human subject protection. In essence, activities arising from this project have in many instances laid down the foundation for the culture of standardised research and evidencebased public health methods in the country.

Lack of training in research methods. A serious challenges in establishing a research infrastructure to support the development and evaluation of tobacco treatment programmes was the lack in training resources in research, and lack of institutional support to scientists who were also poorly integrated within the world's scientific community (Maziak, 2001). For example, graduate or postdoctoral level training is not available for many disciplines from which tobacco scientists typically are drawn, including psychology, epidemiology, public health, and neurosciences. Moreover, medical training in Syria provides little in the way of scientific training in tobacco control, since very few faculties are currently involved in such research. Exacerbating this problem is that lack of opportunities in Syria for research careers causes considerable 'brain drain' with many talented early career scientists leaving the country to seek opportunities elsewhere.

To efficiently address deficits in expertise for both treatment and research, we focused our training efforts on physicians and medical students. By virtue of their scientific training and extensive clinical contact with a large percentage of the smoking population, physicians represent an ideal segment of society to involve in research and treatment capacity-building activities, and involvement of medical students helps to ensure the sustainability of these efforts.

At the SCTS, we provided several forms of training, including on-going mentorship of staff members and trainees, short training periods at collaborating institutions (University of Memphis, Virginia Commonwealth University, and the University of Muenster), and distancelearning and mentorship provided by collaborators in other locations. In addition, the SCTS has sponsored research training workshops in conjunction with major international tobacco conferences, such as the World 
Conference on Tobacco or Health, to support training efforts in the Eastern Mediterranean Region (EMR) beyond Syria and to share our work with the global tobacco control community.

To train physicians and medical students within Syria who had no prior research experience, we primarily used a 'training by doing' model. This training model involves careful task selection, identification of skills required to perform the task, followed by conduction of focused training for the required skills. These iterative learning/application cycles proved to be highly efficient in that trainees could apply their new skills soon after acquisition, receive immediate feedback, and correct and refine their skills on an ongoing basis. This approach provided a successful and cost-effective training model, and is likely to be helpful in other developing country settings where advanced degree academic programmes are not available or not economically feasible.

To enhance our training capacity, we also used distance learning and mentorship. Syrian researchers were connected with expert mentors from the U.S. in their field of interest for a year-long training course. Three Syrian researchers underwent this type of training with a focus on cessation intervention delivery (Asfar, Al Ali, Rastam, Maziak, \& Ward, 2014; Asfar et al., 2008; Ward et al., 2013). This training covered course materials based on Fiore et al. involving a combination of self-readings, homework, report writing, and quizzes (Fiore et al., 2008). These three researchers received on-going mentorship and supervision in tobacco treatment, and became the local experts who provided training and supervision in intervention delivery to physicians involved in several cessation trials, described below.

\section{Challenges for Developing and Testing Culturally appropriate Tobacco Treatment Programmes that can be Integrated in Primary Healthcare Centres in Syria}

The approach selected to develop effective, evidencebased, culturally appropriate tobacco treatment programmes in Syria was to modify validated cessation interventions used in industrialised countries to suit the local setting and target populations, and to evaluate them rigorously using randomised controlled trial (RCT) methodology. Three smoking cessation RCTs have been conducted thus far in Syria, and to our knowledge these are the only cessation RCTs to be conducted and published in EMR.

The first RCT targeted cigarette smokers and was a pilot study tested the effectiveness of culturally tailored intensive behavioural intervention compared to brief intervention (Asfar et al., 2008). The second RCT also targeted cigarette smokers but was a large multi-site, twoarm, placebo controlled, double-blinded trial aimed at testing the effectiveness of NRT for smoking cessation as adjunctive treatment with behavioural counselling (Ward et al., 2013). The third RCT was a pilot, two-arm, parallel group RCT targeted waterpipe smokers and tested the feasibility and efficacy of tailored intensive behavioural intervention compared to brief intervention (Asfar et al., 2014). Below, we review the methods and major findings from each of these RCTs.

Pilot RCT to test the feasibility and efficacy of a culturally tailored intensive behavioural intervention compared to a briefintervention. Because cessation interventions had never been tested or implemented in Syria, as a first step, we conducted a pilot RCT to determine the feasibility and efficacy of a clinic-based behavioural intervention that could easily be implemented in existing health care services (Asfar et al., 2008). Despite evidence that pharmacotherapy increases cessation rates (Fiore et al., 2008) bupropion and similar medications are unavailable in Syria. Therefore, for this pilot trial, we opted to test two intensity levels of behavioural intervention, without pharmacotherapy.

We randomised 50 smokers to either a brief (single session) or intensive (four face-to-face sessions plus six phone follow-ups) hospital-based, free, behavioural counselling intervention. The primary efficacy end point was continues abstinence at three months post-quit day, assessed by self-report and exhaled carbon monoxide levels of $<10$ ppm. Secondary end points were 7 day point-prevalent abstinence and adherence to treatment.

Of the 50 enrolled smokers, mean age was 34.8 years (range 18 to 64 years), 86\% were men, and 64\% married. At the three month follow up, continuous abstinence rates were $8 \%$ and $4 \%$ in the intensive and brief intervention groups, respectively $(p=0.50)$, and 7 -day point-prevalent abstinence rates were $16 \%$ and $4 \%$, respectively $(p=0.34)$ (Asfar et al., 2008). Nicotine dependence predicted abstinence at 3 months. Important perceived barriers to cessation included believing oneself to be dependent on nicotine, lack of access to pharmacotherapy $(72 \%)$, poor social support $(82 \%)$, and waterpipe smoking (16\% of smokers initiated smoking waterpipe during their quit attempt) (Asfar et al., 2008). Adherence in the intensive group was only moderate and was associated with smoking for more years and higher self-efficacy.

The intensive intervention included several widely used behavioural strategies that have been shown to be effective in the U.S. and other high income countries, including stimulus control strategies such as setting a quit date, self-monitoring smoking to increase awareness of smoking patterns, nicotine fading/scheduled smoking, undergoing a 'quit ritual' (getting rid of all smoking products and paraphernalia before the quit attempt), training in problem solving, and coping assistance to support longterm relapse prevention. In addition, several contingency management strategies were used including garnering social support by informing family and friends of the quit attempt to garner social support and encouragement of the use of relaxation techniques and self-reward for successes.

At the 3-month follow-up, a process evaluation was conducted that was very informative about participants' 
perceptions of the helpfulness of these strategies (Asfar et al., 2008). While participants found most strategies helpful, a few were not helpful. Several male participants who informed family and friends of their quit attempt reported being embarrassed to admit when they relapsed. Several men also reported that friends actively attempted to sabotage their quit efforts, usually by offering the cigarettes, which seems to reflect lack of social norms to support cessation efforts. Many participants found the self-monitoring and nicotine fading strategies cumbersome to implement, and were embarrassed when people saw them using monitoring forms and inquired what they were for. Lastly, most participants expressed disappointment, and often surprise, that medication was not offered to help them quit, which seemed to reflect a cultural expectation in Syria that healthcare encounters should result in being given a prescription for medication. These process evaluation findings were used to modify our second cigarette smoking cessation RCT.

A multi-site, two-arm, placebo controlled RCT to test the effectiveness of NRT as adjunctive treatment with behavioural counselling in primary care settings. In our first trial, described above, we did not test pharmacological treatment because cessation medications were not widely available in Syria. However, we decided to use pharmacotherapy combined with face-to-face intervention in our second trial for several reasons. First, our results from our first pilot trial indicated that most smokers have high interest in using medications. Second, most smokers in Syria are heavy smokers and nicotine dependent (Maziak et al., 2004a), and based on tobacco treatment guidelines (Fiore et al., 2008), this treatment is the most effective for this population. Finally, a goal of the SCTS was to encourage government-sponsored primary care clinics to make pharmacotherapy available in Syria. In meeting with the Syrian Minister of Health, we were informed that making pharmacotherapy available in primary care clinics would not be considered unless local data were available to support its effectiveness in Syria. As such, the goal of our second RCT was to evaluate the efficacy of NRT, as well as the feasibility of integrating behavioural cessation counselling in this setting. We selected nicotine patch as the pharmacologic agent since it is easy to use and has few side effects and contraindications.

As noted above, we also modified the behavioural counselling programme in light of lessons learned from the first RCT. Changes included dropping the selfmonitoring/nicotine fading exercise, modifying recommendations for social support enhancement, and increasing 'front loading' of treatment to boost support early in the quitting process.

The trial was conducted in primary care settings due to their ability to reach a large segment of Syria's smoking population. The trial was conducted as an effectiveness study, rather than efficacy study, to increase sustainability by determining whether the intervention could be suc- cessfully delivered in a 'real world' setting. Clinic staffs were trained by physician staff members of the SCTS, who had received extensive tobacco treatment and research experience, as described above.

Two hundred and sixty-nine adult primary care patients 18-65 years of age, who had been smoking continuously for at least one year and were smoking at least five cigarettes per day were recruited from three randomly selected government-supported primary care centres (Ward et al., 2013). One private clinic was also included to ensure broad socioeconomic representation. Primary care physicians at each clinic received in-service training on tobacco epidemiology, health effects, and brief intervention strategies. Clinic-wide procedure were put in place to ensure every patient was screened for smoking, and physicians were instructed to deliver a brief ' $5 \mathrm{~A}$ ' based intervention (Fiore et al., 2008) to ask each patient about their smoking status, advise the patient of the importance of quitting, assess willingness to quit, and refer interested patients to the research team to determine eligibility for the trial.

Enrolled patients received cessation treatment from a specially trained primary care physician who provided behavioural counselling and managed pharmacotherapy. Patients were randomised to receive six weeks of treatment with nicotine versus placebo patch. Primary end-points were prolonged abstinence (no smoking after a 2-week grace period) at end of treatment, and 6 and 12 months post-quit day, assessed by self-report and exhaled carbon monoxide levels of $<10 \mathrm{ppm}$ (Ward et al., 2013).

Approximately 1,000 smokers were treated in the four clinics during the study period. Of these, 426 (43\%) were referred by their physician to assess their eligibility to participate in the study. Of the 426,70 did not meet eligibility criteria, 4 were missed and 83 refused to participate. Thus, $80 \%$ of referred, eligible patients consented to participate and were randomised, with 134 and 135 allocated to nicotine and placebo patch respectively (Ward et al., 2013).

Overall, $82 \%$ of patients were retained through the 12 -month follow-up period $(85 \%$ and $79 \%$ of nicotine and placebo patients, respectively). Adherence was very good, with $94 \%, 84 \%$, and $80 \%$ of patients completing in-person treatment sessions 1, 2, and 3 respectively. Adherence to patch use averaged $93 \%$ and $89 \%$ in the nicotine and placebo groups respectively. Nicotine patch produced expected reductions in urges to smoke and withdrawal symptoms, but no treatment effect was observed. The proportion of patients in the nicotine and placebo groups with prolonged abstinence was $21.6 \%$ and $20.0 \%$, respectively, at end of treatment; $13.4 \%$ and $14.1 \%$ at 6 months; and $12.7 \%$ and $11.9 \%$ at 12 months (all $p$-values > 0.75 ) (Ward et al., 2013).

Pilot RCT to test the efficacy of tailored, intensive behavioural intervention for waterpipe smokers. Because of the rapid spread of waterpipe smoking (Maziak, 2015; Maziak, Ward, Afifi Soweid, \& Eissenberg, 2004b) and 
growing evidence of its adverse health consequences (Akl et al., 2010; Sibai et al., 2014), dependence-producing effects (Eissenberg \& Shihadeh, 2009), and difficulty that many users have in quitting (Ward et al., 2005; Ward, Siddiqi, Ahluwalia, Alexander, \& Asfar, 2015), we developed a behavioural cessation programme for willing-to-quit waterpipe users, and evaluated its feasibility and efficacy in a pilot, two arm, parallel group, randomised, open label trial in Aleppo, Syria. Fifty adults who smoked waterpipe $\geq 3$ times per week for the last year, did not smoke cigarettes, and were interested in quitting were randomised to receive either brief (one in-person session and three phone calls) or intensive (three in-person sessions and five phone calls) behavioural counselling delivered by a trained physician in a clinical setting (Asfar et al., 2014). The primary efficacy end point of the developed interventions was prolonged abstinence at three months post-quit day, assessed by self-report and exhaled carbon monoxide levels of $<10$ $\mathrm{ppm}$. Secondary end points were 7-day point-prevalent abstinence and adherence to treatment.

Of the 50 enrolled waterpipe smokers, 27 were allocated to the intensive arm and 23 to the brief arm. Mean age was 30 years, $94 \%$ were men, and $44 \%$ were married. The proportions of participants in the brief and intensive interventions with prolonged abstinence at the 3-month assessment were $30.4 \%$ and $44.4 \%$, respectively. Previous success in quitting $(\mathrm{OR}=3.57 ; 95 \% \mathrm{CI}=1.03-12.43)$ predicted cessation (Asfar et al., 2014). Higher baseline readiness to quit, more confidence in quitting, and being unemployed predicted a better adherence to treatment. Approximately $30 \%$ of participants were fully adherent to treatment, which did not vary by treatment group.

Overall, $67 \%$ of participants reported that the behavioural intervention was helpful in quitting smoking and $95 \%$ stated that the interventionist was helpful. The most helpful strategies provided by the programme were encouraging physical activity to cope with urges to use waterpipe $(71 \%)$, receiving important educational information $(71 \%)$, using relapse prevention tools (58\%), and getting social support (48\%). While $70 \%$ of participants received support from their families, only $22 \%$ received support from their friends in their quit attempt. These process evaluation data indicate that the programme was generally acceptable and effective, and that very brief intervention (single in person session and a few brief phone follow-up calls) may be as helpful as more intensive behavioural treatment. The fact that only two thirds of participants rated the intervention as helpful indicates that there is considerable room for improvement. One-third of participants were interested in participating in group counselling intervention and $74 \%$ preferred using medication, which should be considered in efforts to improve behavioural treatment of waterpipe smoking.

In summary, results from the three trials indicated that brief behavioural cessation treatment for both cigarette and waterpipe users were feasible and boosted quit rates beyond what would be expected in the absence of treat- ment. However, in contradiction to much of the evidence base developed from rigorous efficacy trials in high income countries, nicotine patches treatment was not effective when given as an adjunct to behavioural support for cigarette cessation. Certainly, the last result need confirmation from other randomised effectiveness trials, particularly as in many low- and middle-income countries, including Syria, NRT is costly and not widely available (Maziak et al., 2004a).

Several challenges were identified during the conduct of our trials. Below we discuss these challenges and lessons learned from our experience:

Coordination of the research activities between the local and main US collaborators. Some of the logistical challenges to this project arose from the fact that two of the main investigators were located in the US, several thousand miles away from where the centre was located. Several mechanisms were taken to ensure the active, regular involvement of all investigators and the smooth operation of all research activities. First, one of the principal investigators on this project was located in Aleppo to oversee all training and research activities in the centre. Second, sufficient funds were allocated to allow American investigators to spend adequate time in Aleppo to guide the training, implementation, and quality assurance of research protocols. Third, Syrian research support staff and trainees spent time in the US at the collaborators' institutions which helped ensure standardised operations and good communication. Regular (i.e., weekly, or more often if necessary) conference calls were held among investigators and research support staff. Finally, sponsoring regional training workshops on a regular basis allowed on-going, close interaction among investigators, and trainees during both planning and implementation of the workshops.

High smoking rates among primary healthcare providers. Before conducting our second trial, all healthcare providers (physicians and nurses) in the participating PHC centres were surveyed to assess their own smoking status, their reported delivery of smoking cessation interventions, and their attitude towered implementing anti-smoking policy. Results revealed that smoking continues to be widespread among physicians and the nursing staff at PHC centres (Asfar, Al-Ali, Ward, Weg, \& Maziak, 2011). More importantly, smoking habits among PHC providers negatively affected providers' willingness and ability to address smoking among their patients and their support to implement tobacco-control policies in PHC centres.

Regarding providers' smoking cessation delivery practices, only half of the participating physicians reported routinely asking patients about their smoking status, and fewer than half of these assisted their patients in their efforts to quit smoking. This missed opportunity may be partially due to limited physician access to resources needed to assist smoking patients; only $5 \%$ of PHC physicians in this study prescribed smoking cessation 
medications. This could indicate either that many PHC physicians in Syria are unaware of these cessation aids or that the availability of these pharmacologic aids is limited in Syria. In addition, most physicians thought that their knowledge was insufficient to help patients quit smoking and were interested in receiving training in cessation counselling. These attitudes may reflect a lack of opportunities for training in this area during medical school or as part of continuing medical education for physicians. Overcoming these challenges will require a national smoking cessation plan that involves training healthcare providers and providing smoking cessation guidelines (Gorin \& Heck, 2004).

Lack of pharmacological agents used in tobacco treatment in Syria. An important challenge for conducting our second behavioural/pharmacological trial was lack of pharmacological agents used in tobacco treatment in Syria. Drugs used in smoking cessation such as NRT or Bupropion were not registered officially in the Syrian health ministry and therefore they are not sold in the country. In order to conduct the study, we obtained the nicotine patches from an American pharmaceutical company. However, our request to obtain the nicotine patches placebo was turned down. This could be explained by the lack of interest of the company in the region mainly because the high price of these drugs may limit market potential for patient in low-income countries. However, our experience in Syria suggests that low income countries may require local evidence of the effectiveness of pharmacological agents to support greater access to these agents. To solve this problem, we relied on a local pharmaceutical company to produce the placebo. The company produced placebo patches identical to the active patches and changed the external cover and the base paper for the active patches to be undistinguishable from the placebo.

Clinical practice guidelines recommend including pharmacological treatment as an adjunct to behavioural cessation counselling given its superior efficacy. Our participants in the three trials expressed high interest in using medication to help them in quitting. Making these medications available in reasonable price is crucial in order to support smokers in their quitting.

Challenges of conducting cessation research in a politically volatile region. Even during the early planning of the SCTS, we confronted numerous obstacles that required adaptation and ever-changing plans (Maziak et al., 2004a). While writing the original NIH grant application that was to fund the SCTS, in the Fall of 2001, the tragedy of 9/11 occurred, temporarily halting all work on the project while we awaited notification from NIH and the U.S. Department of State about whether applications to fund research in the Middle East would be considered. Encouragingly, applications continued to be accepted and we were funded.

New challenges continued to emerge, however, such as difficulty transferring funds into the country, and the temporary shut-down of the SCTS through political meddling from high government officials (e.g., Minister of Health). The most profound and long-lasting challenge has been how to maintain any level of work amidst an on-going four year (and counting) civil war that has displaced millions of Syrians, killed tens of thousands, and destroyed much of the country, including Aleppo, home to the SCTS. Early in the conflict, we were able to maintain productivity due to our committed local staff who stayed in Aleppo. As the situation became increasingly volatile and untenable, staff members were forced to leave Syria, and the SCTS has become a 'virtual' research centre. Our success in forming partnerships with researchers in neighbouring Lebanon and Jordan, as well as several European countries, has allowed the SCTS to adapt (e.g., moving studies to neighbouring countries, conducting regional capacity building) and continue its important mission to advance tobacco control science and capacity in the EMR.

Despite the continued challenges, the SCTS was able to continue to participate effectively in tobacco research and capacity building in the EMR. Prominent on SCTS list of achievements its contribution to several advisories from the World Health Organization and other international health organisations, attainment of numerous NIH and EU grants, and advancing a regional perspective and needs for tobacco control (Maziak et al., 2013). In 2008, the SCTS was recognised by the very prestigious Hamdan Award for the Best Medical Institute in the Arab World.

As tragedy engulfed Syria, SCTS was in a position to study and analyse the human and healthcare costs of the ongoing war, and provide a roadmap to prioritise and guide the rebuilding of the Syrian health care system postconflict (Ben Taleb et al., 2015). But in tobacco control in particular, SCTS's work on the waterpipe has established it as a world leader in generating the evidence base to tackle this growing global epidemic. Data, instruments, and studies from the SCTS have significantly advanced the knowledge base about waterpipe spread, behavioural and social determinants, toxicology, addictiveness, and clinical and policy implications of the waterpipe epidemic. This will remain the lasting impact of this institution on the global tobacco control stage.

\section{Future Directions for Smoking Cessation Efforts in Syria}

Efforts at the SCTS were the first attempt to build evidence on effectiveness of smoking cessation interventions for both cigarettes and waterpipe smokers in Syria. Further studies in this area are still needed to advance this knowledge. Given the high smoking rates in Syria, population-based interventions such as tobacco quitlines have the potential to reduce the burden of tobacco use in the country. Another promising venue to provide smoking cessation intervention is PHC clinics. Many healthcare programmes (e.g., diabetics, family planning, and vaccination) are currently implemented successfully in PHC clinics in Syria. Including smoking cessation intervention within these programmes provide an excellent 
opportunity to reduce smoking among PHC patients. At the individual-level intervention, developing and implementing smoking cessation interventions targeting populations at high risk for smoking such as cancer survivors, patients with cardiovascular and respiratory disease, and pregnant women are urgently needed. Besides cigarette smoking, it is important to respond to the growing epidemic of waterpipe tobacco use, and to continue developing effective and feasible interventions for users who are interested in quitting (Maziak et al., 31 JUL 2015; Ward et al., 2015). The alarming rise of waterpipe smoking among women and youths identify these two groups as high priority targets for future WP smoking cessation interventions. Finally, because substantial numbers of smokers in Syria (16\%) are dual tobacco users (cigarettes and WP) (Ward et al., 2006), smoking cessation interventions targeting dual user also are important.

\section{Conclusions}

Although smoking is a serious problem in Syria, clinical practice standards for smoking cessation are not in place, little work is being done to develop culturally appropriate interventions, and the transferability of effective interventions from more developed nations has not been tested. The SCTS, an NIH-funded pioneer research and capacity building institution in Syria, has built a scientific infrastructure and conducted three RCTs to develop and test culturally adapted smoking cessation interventions in Aleppo, Syria. This review discussed challenges and lesson learned from the SCTS experience. To build a research infrastructure, the first step for the SCTS was to establish the Syria's first IRB, train its members in research ethics and their role/authorities, and provide on-going consultation. The centre also addressed deficits in expertise for both tobacco treatment and research by focusing its training efforts on physicians and medical students. For example, the SCTS provided several forms of training including on-going mentorship of staff members and trainees, short training periods at its international collaborating institutions, distance-learning, and sponsored research training workshops in conjunction with major international tobacco conferences, such as the World Conference on Tobacco or Health. Main challenges to conduct the three tobacco treatment trials were difficulties of coordination between the local and US collaborators, high Smoking Rates among PHC providers, lack of pharmacological agents used in tobacco treatment, and difficulties of conducting research in a politically volatile region. Several strategies were adopted by the SCTS to overcome these challenges. The SCTS ensured an active and regular involvement of all investigators, and advocated for a national smoking cessation plan that involves training healthcare providers in smoking cessation treatment and make pharmacological agents used in smoking cessation available. Further studies are needed to develop and test smoking cessation interventions at the population level (e.g., tobacco quit- lines, PHC), and individual level. Mainly, populations at high risk for smoking such as cancer survivors, patients with cardiovascular and respiratory disease, and pregnant women, should be targeted for such interventions. Finally, waterpipe users, particularly women and youths, and dual tobacco users (cigarettes and waterpipe) are high priority groups for smoking cessation efforts.

\section{Acknowledgements}

TA and KDW were responsible for the study concept and design. TA wrote the first draft of the manuscript, and all authors participated in critically revising and editing the manuscript. All authors have approved the final manuscript.

\section{Financial Support}

This work is supported by NIH Grant \# R01DA035160 (P.I. Dr. Maziak).

\section{Conflict of Interest}

All authors confirm that we have no financial or other conflicts of interest.

\section{References}

Akl, E. A., Gaddam, S., Gunukula, S. K., Honeine, R., Jaoude, P. A., \& Irani, J. (2010). The effects of waterpipe tobacco smoking on health outcomes: A systematic review. International Journal of Epidemiology, 39(3), 834-857.

Asfar, T., Al Ali, R., Rastam, S., Maziak, W., \& Ward, K. D. (2014). Behavioral cessation treatment of waterpipe smoking: The first pilot randomized controlled trial. Addictive Behaviors, 39(6), 1066-1074.

Asfar, T., Al-Ali, R., Ward, K. D., Weg, M. W. V., \& Maziak, W. (2011). Are primary health care providers prepared to implement an anti-smoking program in Syria? Patient Education and Counseling, 85(2), 201-205. doi:10.1016/j.pec.2010.11.011.

Asfar, T., Ward, K. D., Eissenberg, T., \& Maziak, W. (2005). Comparison of patterns of use, beliefs, and attitudes related to waterpipe between beginning and established smokers. BMC Public Health, 5(1), 19.

Asfar, T., Weg, M. V., Maziak, W., Hammal, F., Eissenberg, T., \& Ward, K. D. (2008). Outcomes and adherence in Syria's first smoking cessation trial. American Journal of Health Behavior, 32(2), 146-156. doi:10.5555/ajhb.2008.32.2.146.

Ben Taleb, Z., Bahelah, R., Fouad, F. M., Coutts, A., Wilcox, M., \& Maziak, W. (2015). Syria: Health in a country undergoing tragic transition. International Journal of Public Health, 60(Suppl. 1), S63-S72. doi:10.1007/s00038-014-0586-2.

Control, C. f. D., \& Prevention. (2011). Quitting smoking among adults-United States, 2001-2010. MMWR. Morbidity and Mortality Weekly Report, 60(44), 1513.

Eissenberg, T., \& Shihadeh, A. (2009). Waterpipe tobacco and cigarette smoking: Direct comparison of toxicant exposure. American Journal of Preventive 
Medicine, 37(6), 518-523. doi:S0749-3797(09)00583-2 [pii] 10.1016/j.amepre.2009.07.014.

Fiore, M. C., Jaén, C. R., Baker, T. B., Bailey, W. C., Benowitz, N. L., Curry, S. J. et al. (2008). Treating tobacco use and dependence: 2008 update. Clinical practice guideline. Rockville, MD: US Department of Health and Human Services.

Gorin, S. S., \& Heck, J. E. (2004). Meta-analysis of the efficacy of tobacco counseling by health care providers. Cancer Epidemiology Biomarkers \& Prevention, 13(12), 20122022.

Jha, P., \& Chaloupka, F. J. (2000). The economics of global tobacco control. BMJ: British Medical Journal, 321(7257), 358.

Maziak, W. (2001). Global health research. Science aid programme could be set up to help researchers in developing countries [letter]. BMJ. British Medical Journal, 322(7279), 173.

Maziak, W. (2002). Smoking in Syria: Profile of a developing Arab country. The International Journal of Tuberculosis and Lung Disease, 6(3), 183-191.

Maziak, W. (2015). Rise of waterpipe smoking. BMJ, 350, h1991.

Maziak, W., Arora, M., Reddy, K., \& Mao, Z. (2006). On the gains of seeding tobacco research in developing countries. Tobacco Control, 15(Suppl. 1), i3-i4.

Maziak, W., Eissenberg, T., Klesges, R., Keil, U., \& Ward, K. (2004a). Adapting smoking cessation interventions for developing countries: A model for the Middle East. The International Journal of Tuberculosis and Lung Disease, 8(4), 403-413.

Maziak, W., Jawad, M., Jawad, S., Ward, K. D., Eissenberg, T., \& Asfar, T. (31 Jul 2015). The Cochrane Review: Interventions for waterpipe smoking cessation. doi: 10.1002/14651858.CD005549.pub3.

Maziak, W., Nakkash, R., Bahelah, R., Husseini, A., Fanous, N., \& Eissenberg, T. (2013). Tobacco in the Arab world: Old and new epidemics amidst policy paralysis. Health Policy and Planning, czt055. doi:10.1093/heapol/czt055.

Maziak, W., Ward, K. D., Afifi Soweid, R. A., \& Eissenberg, T. (2004b). Tobacco smoking using a waterpipe: A re-emerging strain in a global epidemic. Tobacco Control, 13(4), 327-333. doi:13/4/327 [pii] 10.1136/tc.2004.008169

Maziak, W., Ward, K. D., Eissenberg, T., Klesges, R. C., \& Keil, U. (2004c). The Syrian center for tobacco studies: A model of international partnership for the creation of sustainable research capacity in developing countries. Global Health Promotion, 11(2), 93.

Richmond, R., \& Heather, N. (1990). General practitioner interventions for smoking cessation: past results and future prospects. Behaviour Change, 7(03), 110-119.

Sibai, A. M., Tohme, R. A., Almedawar, M. M., Itani, T., Yassine, S. I., Nohra, E. A. et al. (2014). Lifetime cumulative exposure to waterpipe smoking is associated with coronary artery disease. Atherosclerosis, 234(2), 454-460. doi:10.1016/j.atherosclerosis.2014.03.036.

The war on Syrian civilians. (2014). Lancet, 383(9915), 383. doi:10.1016/S0140-6736(14)60134-3.

Ward, K. D., Asfar, T., Al Ali, R., Rastam, S., Weg, M. W. V., Eissenberg, T. et al. (2013). Randomized trial of the effectiveness of combined behavioral/pharmacological smoking cessation treatment in Syrian primary care clinics. Addiction, 108(2), 394-403.

Ward, K. D., Eissenberg, T., Rastam, S., Asfar, T., Mzayek, F., Fouad, M. F. et al. (2006). The tobacco epidemic in Syria. Tobacco Control, 15(Suppl. 1), i24-i29. doi:15/suppl_1/i24 [pii] 10.1136/tc.2005.014860.

Ward, K. D., Hammal, F., VanderWeg, M. W., Eissenberg, T., Asfar, T., Rastam, S. et al. (2005). Are waterpipe users interested in quitting? Nicotine Tob Res, 7(1), 149-156. doi:U03T810711538J26 [pii] 10.1080/ 14622200412331328402 .

Ward, K. D., Siddiqi, K., Ahluwalia, J. S., Alexander, A. C., \& Asfar, T. (2015). Waterpipe Tobacco smoking: The critical need for cessation treatment. Drug and Alcohol Dependence, $153,14-21$.

WHO. (2008). WHO REPORT on the global TOBA CCO epidemic, 2008. The MPOWER package. Retrieved from http://www.who.int/tobacco/mpower/mpower_report_ full_2008.pdf. 